\title{
artigo
}

\section{Importância da assistência farmacêutica na estratégia saúde da família em unidades de saúde do município de Caruaru-PE}

Importance of pharmaceutical assistance in the family health strategy in health units in the municipality of Caruaru-PE Importancia de la asistencia farmacéutica en la estrategia de salud familiar en las unidades de salud del municipio de Caruaru-PE

\section{RESUMO}

O presente estudo objetivou demonstrar a importância da Assistência Farmacêutica na Estratégia Saúde da Família (ESF) no ponto de vista de usuários assistidos em dez unidades de saúde do município de Caruaru-PE. A coleta de dados foi realizada por meio de um questionário, permitindo traçar o perfil dos pacientes que adquiriam seus medicamentos nas unidades do município. Foi observada baixa renda familiar per capita (53\%) e baixa escolaridade $(73,5 \%)$ na população estudada, o que representa fatores agravantes na efetividade da farmacoterapia bem como o acesso aos medicamentos. Na análise da adesão ao tratamento farmacológico, $57 \%$ dos usuários afirmaram ter dificuldades para completar seu tratamento dada a falta de medicamentos nas unidades. 91,6\% dos entrevistados acreditam que a presença do profissional farmacêutico na ESF pode garantir maior acesso dos usuários aos medicamentos bem como o uso racional dos mesmos, além de contribuir para a valorização e aperfeiçoamento do serviço farmacêutico assistencial.
\end{abstract}

DESCRITORES: Assistência Farmacêutica; Estratégia Saúde da Família e Uso de Medicamentos.

\begin{abstract}
This study aimed to demonstrate the importance of Pharmaceutical Assistance in the Family Health Strategy (FHS) from the point of view of users assisted in ten health units in the municipality of Caruaru-PE. Data collection was carried out through a questionnaire, allowing the profile of patients who purchased their drugs at units in the city to be drawn. Low per capita family income (53\%) and low education (73.5\%) were observed in the studied population, which represents aggravating factors in the effectiveness of pharmacotherapy as well as access to medicines. In the analysis of adherence to pharmacological treatment, $57 \%$ of users said they had difficulties completing their treatment due to the lack of medication in the units. $91.6 \%$ of the interviewees believe that the presence of the pharmaceutical professional in the FHS can guarantee greater access for users to medicines as well as their rational use, in addition to contributing to the enhancement and improvement of the pharmaceutical assistance service.
\end{abstract}

DESCRIPTORS: Pharmaceutical Services; Family Health Strategy and Drug Utilization

\section{RESUMEN}

Este estudio tuvo como objetivo demostrar la importancia de la Asistencia Farmacéutica en la Estrategia de Salud Familiar (ESF) desde el punto de vista de los usuarios atendidos en diez unidades de salud del municipio de Caruaru-PE. La recogida de datos se realizó a través de un cuestionario, lo que permitió trazar el perfil de los pacientes que adquirieron sus medicamentos en unidades de la ciudad. En la población estudiada se observaron bajos ingresos familiares per cápita (53\%) y baja escolaridad $(73,5 \%)$, lo que representa factores agravantes en la efectividad de la farmacoterapia y en el acceso a medicamentos. En el análisis de adherencia al tratamiento farmacológico, el 57\% de los usuarios manifestó haber tenido dificultades para completar su tratamiento debido a la falta de medicación en las unidades. El 91,6\% de los entrevistados cree que la presencia del profesional farmacéutico en la ESF puede garantizar un mayor acceso de los usuarios a los medicamentos así como su uso racional, además de contribuir a la potenciación y mejora del servicio de asistencia farmacéutica.

DESCRIPTORES: Servicios Farmacéuticos; Estrategia de Salud Familiar y Utilización de Medicamentos.

RECEBIDO EM: 15/09/2020 APROVADO EM: 30/09/2020 


\section{Jucélia Ivonete dos Santos}

Farmacêutica e Mestranda em Gestão, Inovação e Consumo (PPGIC), Universidade Federal de Pernambuco-UFPE. Caruaru-PE, Brasil. Pós-Graduada em Farmácia Hospitalar e Clínica, Centro Universitário Redentor, Recife-PE, Brasil.

ORCID: 0000-0003-2375-0456

\section{Valéria da Silva Tabosa}

Farmacêutica, Pós-Graduada em Farmácia Hospitalar e Clínica, Centro Universitário Redentor, Recife-PE, Brasil. ORCID: 0000-0003-0277-6438

\section{Ákylla Fernanda Souza Silva}

Farmacêutica e Mestranda em Biologia Celular e Molecular Aplicada, pela Universidade de Pernambuco-UPE, Recife-PE, Brasil. ORCID: 0000-0002-5186-8227

\section{Carlos Eduardo Miranda de Sousa}

Graduado em Farmácia, Universidade Federal Pernambuco- UFPE, Mestrado em Ciências Farmacêuticas e Doutorado em Ciências Farmacêuticas, Universidade Federal Pernambuco- UFPE. Professor e Supervisor de estágio do Centro Universitário de Santo Antão.

ORCID: 0000-0003-0959-5912

\section{INTRODUÇÃO}

0 Sistema Único de Saúde (SUS) foi o primeiro modelo brasileiro de sistema de saúde a adotar a Assistência Farmacêutica (AF), possibilitando ao farmacêutico participar de maneira mais efetiva da saúde pública e desenvolver formas específicas de tecnologias envolvendo os medicamentos e a prestação de serviços de saúde ${ }^{(1)}$. AF é definida como sendo "um conjunto de ações voltadas à promoção, proteção e recuperação da saúde, tanto individual como coletiva, tendo o medicamento como insumo essencial" (2).

No âmbito do Sistema Único de Saúde (SUS), a AF tem como objetivo primordial garantir o abastecimento contínuo e o uso racional de medicamentos nas Unidades Básicas de Saúde, mediante processos de seleção, programação, aquisição, armazenamento, prescrição e dispensação ${ }^{(3)}$. As leis $8080 / 90$ e $8142 / 90$ consagram os princípios da descentralização dos serviços de saúde e municipalização da gestão, destacando o artigo $6^{\circ}$ da Lei $n^{\circ} 8080$ que "assegura o provimento da Assistência Tera-pêutica integral, incluindo a Assistência Farmacêutica” ${ }^{(1)}$.

Com a descentralização das ações de saúde, os municípios passaram a assumir a responsabilidade direta pela atenção à saúde, incluindo as ações relacionadas à $\mathrm{AF}$ através do Programa Saúde da família, que

\section{Dentre os serviços}

prestados à

população nas

unidades de saúde

encontra-se a

distribuição de

medicamentos,

que visa facilitar $o$

acesso da população

aos medicamentos

essenciais. surgiu como estratégia para a reorientação do antigo modelo assistencial, sendo posteriormente transformado em Estratégia Saúde da Família $(\mathrm{ESF})^{(4)}$. As equipes que compõem essa estratégia, denominadas Equipes de Saúde da Família, são compostas por médico generalista ou médico de família, enfermeiro, auxiliar de enfermagem e Agentes Comunitários de Saúde ${ }^{(5)}$.

Dentre os serviços prestados à população nas unidades de saúde encontra-se a distribuição de medicamentos, que visa facilitar o acesso da população aos medicamentos essenciais ${ }^{(6)}$. Com a prática de distribuição de medicamentos em unidade da ESF, surge a necessidade de se garantir maior controle sanitário destes medicamentos, com base na RDC n $328 / 99$, que dispõe sobre as boas práticas de dispensação para farmácia e drogaria e assegura que o profissional farmacêutico seja o responsável pela supervisão do conjunto de processos que caracteriza a $\mathrm{AF}^{(7)}$.

$A$ incorporação do farmacêutico às ESF torna-se uma medida essencial para garantia da AF, podendo reduzir possíveis os problemas relacionados ao tratamento dos pacientes, alcançando resultados definidos para a melhoria da sua qualidade de vida (8).No entanto,apesar de sua importância na composição das ESF, a atuação do farmacêutico nas unidades básicas de saúde ainda é uma realidade pouco comum.

Assim, o presente estudo tem como 
objetivo demonstrar a importância do farmacêutico na ESF no ponto de vista dos usuários que são por ela assistidos, que adquirem medicamentos nas unidades básicas de saúde do município de Caruaru, Pernambuco, região Nordeste do Brasil.

\section{MÉTODO}

O estudo é caracterizado como transversal descritivo de base populacional, realizado com usuários de dez unidades básicas de saúde assistidos pela SF na zona urbana de um município situado no interior do Estado de Pernambuco, entre os meses de janeiro de 2016 a dezembro de 2017. A coleta de dados foi realizada por meio de um questionário pré-codificado, previamente testado. Foram coletadas informações quanto ao sexo, idade, renda familiar per capita, histórico de doenças crônicas referidas pelo indivíduo, posse de plano privado de saúde, práticas de trabalho dos profissionais que dispensam medicamentos nas unidades e conhecimentos gerais sobre AF na ESF.

O universo amostral foi composto por 200 usuários em 10 unidades de saúde, sendo uma unidade por bairro. Foram entrevistados de 5-10\% dos usuários, de acordo com o número de atendimento médico mensal em cada unidade que, segundo a Secretaria de Saúde do município, o número de atendimentos é de 250 a 550/mês, variando de unidade para unidade. Participaram da pesquisa, indivíduos maiores de idade que fizesse parte da área de cobertura da UBS, e que adquirissem seus medicamentos na unidade abordada. Foram excluídos da pesquisa, pessoas com alguma dificuldade para responder o protocolo

\section{Tabela 1. Informações sobre os serviços farmacêuticos assistenciais.}

\section{Questões}

Já ouviu falar nos serviços farmacêuticos assistenciais

Já foi orientado por um profissional farmacêutico

Os serviços farmacêuticos contribuiriam para o URM

*URM- Uso Racional de Medicamentos

*NS/NR - Não sabe/Não Respondeu
Os profissionais

apontados na

pesquisa como

sendo responsáveis

pela dispensação

dos medicamentos

nas unidades, $45 \%$

eram enfermeiros,

$44,5 \%$ técnicos de

enfermagem e 8,5\%

Agente Comunitário

de Saúde.

(Ex: doenças psíquicas ou neurológicas) e que não estivesse com um acompanhante maior de idade que possa responder por elas. Os dados foram armazenados em um banco de dados utilizando o aplicativo Microsoft Excel, sendo calculado e obtido o percentual das variáveis para cada seção do questionário aplicado. A pesquisa foi iniciada após aprovação pelo Comitê de Ética em Pesquisa (CEP), do Centro Universitário Tabosa de Almeida (ASCES-UNITA), sob o ofício n ${ }^{\circ} 1.821 .970$ e CAAE 60885916.4.0000.5203.

\section{RESULTADOS}

A análise da efetividade da farmacoterapia dos usuários assistidos pela ESF, realizada através dos dados socioeconômicas e culturais da população, apresentou dados preocupantes no que se refere a orientação terapêutica e acesso aos fármacos. Os resultados obtidos demonstraram que $73,5 \%$ dos entrevistados tinham baixa escolaridade e que $53 \%$ possuíam renda familiar per capita menor ou igual a um salário mínimo.

Avaliando o perfil de distribuição de medicamentos nas unidades de saúde, os resultados demonstraram um índice elevado de usuários dependentes dos medicamentos distribuídos na UBS, equivalente a 98\% onde, desses, 93\% fazem aquisições mensais dos mesmos medicamentos. Quanto à adesão ao tratamento farmacológico, os resultados demonstraram que $17,24 \%$ dos usuários interrompem o tratamento após melhora do seu estado clínico.

Os profissionais apontados na pesquisa como sendo responsáveis pela dispensação dos medicamentos nas unidades, $45 \%$ eram enfermeiros, $44,5 \%$ técnicos de enfermagem e 8,5\% Agente Comunitário de Saúde. Em nenhuma unidade foi apontada a presença do farmacêutico exercendo tal função, e em nenhuma das unidades havia sequer a presença desse profissional compondo a ESF. Parte dos entrevistados não conhecia os serviços farmacêuticos assistenciais e afirmaram nunca terem sido orientados por um farmacêutico, o que demonstra que as atribuições desse profissional na ESF ainda é uma realidade pouco conhecida (Tabela 1).

\section{DISCUSSÃO}

As condições socioeconômicas e culturais de uma dada população, bem como o nível de escolaridade e renda familiar per capita, exige dos médicos e profissionais responsáveis pela orientação e dispensação das medicações, uma maior dedicação à informação dada ao paciente, a fim de garantir o êxito do tratamento (9). De um
Variáveis $(\%)$

SIM NÃO NS/NR

$25,5 \quad 73 \quad 1,5$

$17 \quad 80$

$91,6 \quad 7,4 \quad 1$


modo geral, os medicamentos representam as intervenções terapêuticas de maior relação custo-efetividade, quando prescritos e administrados de forma racional. Os medicamentos destinados a atenção básica são considerados ainda mais importantes por atender às prioridades em saúde da população ${ }^{(10)}$. Assim, a falta de medicamentos no setor público torna-se preocupante uma vez que obriga ao usuário do SUS buscar em farmácias privadas medicamentos considerados essenciais, comprometendo a renda do paciente e consequentemente o seu tratamento ${ }^{(11)}$.

O abandono terapêutico é um grande desafio para a efetividade da farmacoterapia, podendo gerar o agravamento do estado de saúde do paciente ${ }^{(12)}$. Para Bovo et al ${ }^{(13)}$ um outro grande problema en- frentado no sistema público está no ato da dispensação dos medicamentos, onde, em seu estudo, ele pode observar que $64 \%$ dos usuários não recebiam nenhuma orientação. De acordo com a RDC n 328/99, o farmacêutico é o profissional responsável pela supervisão da dispensação de medicamentos e possui conhecimento científico específico para tal atividade ${ }^{(7)}$.

Em um estudo realizado por Canabarro e Hahn ${ }^{(6)}$ demonstrou que em 9, de 15 unidades de saúde, o enfermeiro era o profissional responsável pelo estoque de medicamentos, e que em 11 equipes o médico era quem distribuía os medicamentos aos pacientes, orientando-os em todos casos. No entanto, é importante lembrar que o farmacêutico se encontra na interface entre a distribuição dos medicamentos e o seu uso, podendo identificar, corrigir ou reduzir possíveis riscos associados a terapêutica ainda dentro do sistema de saúde ${ }^{(15,16)}$.

\section{CONCLUSÃO}

Tendo em vista a deficitária distribuição de medicamentos nas unidades básicas de saúde estudadas, a inserção do farmacêutico é fundamental para a garantia do acesso aos medicamentos e seu uso racional, contribuindo para a valorização e aperfeiçoamento do serviço farmacêutico assistencial. Assim, cabe aos órgãos competentes e profissionais que compóem a ESF analisar a eficiência desta estratégia enquanto distribuidora de medicamentos, mediante a revisão do conjunto de processos que engloba a AF. -

\section{REFERÊNCIAS}

1. Guidoni CM, Olivera CMX, Freitas OL, Pereira RL. Assistência ao diabetes no Sistema Único de Saúde: análise do modelo atual. BrazilianJournalofPharmaceuticalSciences vol. 45, n. 1, jan./mar., 2009.

2. Brasil. Resolução CNS no 338/2004. Aprova a Política Nacional de Assistência Farmacêutica. [acessado em 2017]. Disponível em: http//www.saude.gov.br/doc/ resolucaocns338/cns.htm Acesso: 22 de agosto de 2014.

3. Marques LAM, Vale FVVR, Nogueira VAS, Mialhe FL, Silva LC. Atenção farmacêutica e práticas integrativas e complementares no SUS: conhecimento e aceitação por parte da população são joanense, Physis Revista de Saúde Coletiva, Rio de Janeiro, 21 [ 2 ]: 663-674, 2011.

4. Rover MRM, Manzini F, Farias MR, Mendes SJ, Leite SN. Gestão da Assistência Farmacêutica: avaliação de um município catarinense. Universidade Federal DE Santa Catarina Belo Horizonte, 2013.

5. Andrade LOM. A estratégia Saúde da Família. In: Duncan BB, Schmidt MI, Giugliani ERJ. Medicina ambulatorial: condutas de atenção primária baseadas em evidências. Porto Alegre: Artmed; 2004. p. 88-99.

6. Canabarro IM, Hahn S. Panorama da assistência farmacêutica na saúde da família em município do interior do estado do Rio Grande do Sul. Epidemiologia e Serviços de Saúde, v. 18, n. 4, p. 345-355, 2009.

7. Brasil. Agência Nacional de Vigilância Sanitária. Resolução $n^{\circ}$ 328 , de 22 de julho de 1999. Dispõe sobre requisitos exigidos para a dispensação de produtos de interesse à saúde em farmácias e drogarias [Internet]. Diário Oficial da União, Brasília, p. 14, 26 jul. 1999. Seção 1 [acessado em 2017]. Disponivel em: http:// anvisa.gov.br/legis/resol/328_99.htm.
8. Conselho Federal de Farmácia. Proposta de estruturação da assistência farmacêutica na atenção básica de saúde com a inserção do farmacêutico [Internet]. Brasília: CFF [acessado em 2017]. Disponível em: http://www.cff.org.br.

9. Lima GB, Nunes LCC, Barros JAC. Uso de medicamentos armazenados em domicílio em uma população atendida pelo Programa Saúde da Família. Ciênc. saúde coletiva, v. 15, n. 3, p. 3517-22, 2010.

10. World Health Organization (WHO). Selección de medicamentosesenciales. In: Perspectivas políticas sobre medicamentos de la OMS 4:1-6, 2002.

11. Portela AS, Leal AAF, Werner RPB, Simões MOS, Medeiros ACD. Políticas públicas de medicamentos: trajetória e desafios. RevCiêncFarm Básica Apl., 2010;31(1):09-14 ISSN 1808-4532.

12. Dias CRC, Romano-Lieber NS. Processo de implantação da política de medicamentos genéricos no Brasil. Cad. Saúde Pública. 2006; 22(8):1661-1669.

13. Bovo F, Wisniewski $P$, Morskei MLM. Atenção Farmacêutica: papel do farmacêutico na promoção da saúde Pharmaceuticalcare: role ofpharmacistonpromotionofhealth. Biosaúde, Londrina, v. 11, n. 1, p. 43-56, jan./jun. 2009.

14. Nicoline CB, Vieira RCPA. Assistência farmacêutica no Sistema Único de Saúde (SUS): percepções de graduandos em Farmácia. Interface - Comunic. 2008.

15. Pepe VLE, Castro CGSO. A interação entre prescritores, dispensadores e pacientes: informação compartilhada como possível benefício terapêutico, 2000.

16. Cipolle RJ, Strand LM, Morley PC. O exercício do cuidado farmacêutico. Brasília: Conselho Federal de Farmácia, 2006. 